\title{
Synapses in neurodegenerative diseases
}

\author{
Jae Ryul Bae ${ }^{1}$ E Sung Hyun Kim ${ }^{2, *}$ \\ ${ }^{1}$ Department of Biomedical Science, Graduate School, Kyung Hee University, ${ }^{2}$ Department of Physiology, School of Medicine, Kyung Hee \\ University, Seoul 02447, Korea
}

\begin{abstract}
Synapse is the basic structural and functional component for neural communication in the brain. The presynaptic terminal is the structural and functionally essential area that initiates communication and maintains the continuous functional neural information flow. It contains synaptic vesicles (SV) filled with neurotransmitters, an active zone for release, and numerous proteins for SV fusion and retrieval. The structural and functional synaptic plasticity is a representative characteristic; however, it is highly vulnerable to various pathological conditions. In fact, synaptic alteration is thought to be central to neural disease processes. In particular, the alteration of the structural and functional phenotype of the presynaptic terminal is a highly significant evidence for neural diseases. In this review, we specifically describe structural and functional alteration of nerve terminals in several neurodegenerative diseases, including Alzheimer's disease (AD), Parkinson's disease (PD), Amyotrophic lateral sclerosis (ALS), and Huntington's disease (HD). [BMB Reports 2017; 50(5): 237-246]
\end{abstract}

\section{INTRODUCTION}

Synapse is a fundamental unit for brain function. Flow of neural information between neurons is ignited from presynaptic terminals by the release of a small chemical ingredient called neurotransmitter. This is stored in the small endosomal compartment (synaptic vesicle (SV)), and it's released by the fusion of SV, facilitated by a series of neuronal-activity triggered action of molecular players in the release area (active zone) at the presynaptic terminals.

The presynaptic terminal is composed of several structural and functional components. Cytomatrix proteins (Bassoon and Piccolo) and cytoskeletal protein (actin) provide the structural framework. The active zone (AZ) is the critical site for neurotransmitter release. Many essential molecular machineries

*Corresponding author. Tel: +82-2-961-0286; Fax: +82-2-9642195; E-mail: sunghyunkim@khu.ac.kr

https://doi.org/10.5483/BMBRep.2017.50.5.038

Received 7 March 2017

Keywords: Alzheimer's disease, Neurotransmission, Parkinson's disease, Presynaptic terminals, Synaptic vesicle are localized in the AZ, such as SNARE components for fusion, voltage-gated $\mathrm{Ca}^{2+}$ channels, cell adhesion molecules, etc. Each nerve terminal possesses around 100-200 synaptic vesicles. The synaptic vesicle (SV), a tiny endosomal compartment ( $\sim 40 \mathrm{~nm}$ diameter), contains the neurotransmitter which associates directly and/or indirectly with more than a hundred proteins for proper functioning. For appropriate physiological functions, a number of proteins exist at the nerve terminals. Physiologically, the regulation and maintenance of neurotransmitter release remain critical questions. Several distinct SV pools distributed in the presynaptic terminal and $\mathrm{SV}$ exocytosis is tightly regulated by $\mathrm{Ca}^{2+}$ and its molecular players. Subsequently, SV retrieval continuously maintains the synaptic communication via several endocytic pathways. However, the morphological and physiological intact is easily altered in various neurological diseases. From synaptic vesicle and synaptic protein depletion to neurotransmission and $\mathrm{Ca}^{2+}$ dynamics impairment, a number of alterations in the structure and function of nerve terminals are exhibited in neurological diseases. Furthermore, these presynaptic dysfunctions are thought to be the very early symptoms of neuronal disorders.

This review specifically describes the structural and functional presynaptic alterations in neurodegenerative diseases. Alzheimer's disease (AD) is a high impact neurodegenerative disease. Several pathogenic factors have been identified, such as amyloid beta (A $\beta$ ) plaque, neurofibrillary tangle, and ApoE4. However, the exact pathological etiology requires to be further explored. It is important to understand synaptic alterations by these factors at the initial stage, before the eventual occurrence of neuronal cell death. Parkinson's disease (PD) is the second most common neurodegenerative disease, exhibiting degeneration of dopaminergic neurons in the substantia nigra pars compacta. Consequently, this results in dopamine depletion in the brain, leading to several neurological symptoms, tremors, bradykinesia, and rigidity. A number of sporadic and familial factors have been discovered. Some of the evidences have reported that these factors are deeply implicated with presynaptic function, although it is still much less known how PD is initiated. Other neurodegenerative diseases, such as Huntington disease (HD) and amyotrophic lateral sclerosis (ALS), are also involved in synaptic dysfunction. We describe in depth normal and pathological phenotype of these factors at presynaptic terminals.

ISSN: 1976-670X (electronic edition)

Copyright (c) 2017 by the The Korean Society for Biochemistry and Molecular Biology

(c) This is an open-access article distributed under the terms of the Creative Commons Attribution Non-Commercial License (http://creativecommons.org/licenses/by-nc/4.0) which permits unrestricted non-commercial use, distribution, and reproduction in any medium, provided the original work is properly cited. 


\section{NERVE TERMINALS IN ALZHEIMER'S DISEASE}

Alzheimer's disease (AD), the most common type of dementia, is a rapidly emergent and prominent neurodegenerative disease. The patient progressively loses their memory with a decline in cognition, eventually reaching mortality due to death of the brain cells. Several causative genetic factors have been revealed. Oligomerization of the amyloid beta (A $\beta$ ) plaque from amyloid precursor protein (APP) by BACE and $\gamma$-secretase, is a well-known factor for AD. Mutation or modification of the Tau protein aggregates to form neurofibrillary tangle (NFT) or paired helical filaments (PHF), called Tauopathy, which is also a known cause of AD. A critical genetic factor for late-onset $A D$ is apolipoprotein $E$, particularly $\varepsilon 4$ isoforms (ApoE4). Although these genetic factors are identified and characterized, a number of complicities are still emerging, and remain elusive. Here, we describe the genetic factors involved in the function and dysfunction at the presynaptic terminals (Table 1).

\section{Amyloid precursor protein (APP) and Amyloid beta (A $\beta)$}

Amyloid precursor protein (APP) is an essential source for amyloid beta 40 or 42 (A $\beta 40$ or 42 ) which are known major pathogenic factors in AD. APP normally participates in presynaptic function, although primary function of APP is still not explored. APP is enriched in nerve terminals with Rab5 positive large vesicular organelle (1) or a small set of synaptic vesicles (2), and is involved in structure and function of nerve terminals. APP modulates the initial nerve terminal formation. Cultured neurons from APP knock-out brain revealed upregulation of synaptophysin, a presynaptic marker. Consistently, immunohistochemistry from a slice of APP KO brain showed a high intensity of synaptophysin, indicating that APP is a negative regulator of synaptic formation. Secondly, APP is also involved in physiological modulation of synaptic functions.
APP KO neurons significantly increase the readily releasable pool (RRP) of synaptic vesicles (3). According to computational analysis of APP, it is likely to serve as a hub protein in the presynaptic active zone (PAZ), and is a context regulator in the hippocampal active zone network (4).

Amyloid beta $(A \beta)$ is a fragment peptide from APP, cleaved by BACE and $\gamma$-secretase. Oligomeric aggregation of $A \beta$ peptide is a critical pathogenic factor in AD. Several reports exhibit the $A \beta$ tangle effects in nerve terminal phenotype. Treatment of $A \beta$ oligomer in neurons results in significantly decreasing the presynaptic protein expression, but not post-synapse (5), indicating that $A \beta$ affects the initial structural formation of presynaptic terminals. Physiologically soluble $A \beta$ binds to APP, inducing the APP-APP homodimer. Consequently, there is a boost of $\mathrm{Ca}^{2+}$ influx, eventually resulting in an increase in the release probability $(6,7)$, indicating that $A \beta$ is a positive regulator of neurotransmission at nerve terminal. However, in pathological conditions, increased $A \beta$ can perturbs the release probability by altering spike probability of neurons (8). Internalized $A \beta$ is localized at the nerve terminal, subsequently disrupting the synaptic vesicle protein VAMP2 function for vesicle fusion (9). In addition, it induces the depletion of presynaptic mitochondria and decreases its motility, thereby decreasing the size of synaptic vesicle pool.

Another important feature of the $A \beta$ at synapses is that the synaptic activity for neurotransmission and release of $A \beta$ is tightly correlated, and a nerve terminal is a major place for $A \beta$ release. The brain interstitial fluid (ISF) reveals that synaptic activity influences the $A \beta$ levels. The more the synaptic activity, higher is the $A \beta$ level in ISF. This result correlates with APP endocytosis. The cleavage of APP to produce A $\beta$ occurs in the endosomes or a small fraction of $\mathrm{SV}$, and not on the surface of the plasma membrane (2). Synaptic vesicle exocytosis is thus required for more endocytosis of APP.

Table 1. Summary of presynaptic phenotype by AD genetic factors

\begin{tabular}{|c|c|c|}
\hline \multicolumn{3}{|c|}{ Alzheimer's disease } \\
\hline Factor & Phenotype at presynaptic terminal & Ref \\
\hline $\begin{array}{l}\text { Amyloid Precursor } \\
\text { Protein (APP) }\end{array}$ & $\begin{array}{l}\text { - Negative regulator of synapse formation } \\
\text { - Negative regulator for readily releasable pool of synaptic vesicle } \\
\text { - Molecular Hub in presynaptic active zone (PAZ) }\end{array}$ & $\begin{array}{l}3 \\
3 \\
4\end{array}$ \\
\hline $\begin{array}{l}\text { Amyloid beta } \\
(\mathrm{A} \beta)\end{array}$ & $\begin{array}{l}\text { - Downregulation of presynaptic protein expression } \\
\text { - Increase release probability (soluble A } \beta \text { - normal condition) } \\
\text { - Disruption of vesicle fusion ability by inhibiting VAMP2 function (pathologic A } \beta \text { ) }\end{array}$ & $\begin{array}{l}5 \\
7 \\
9\end{array}$ \\
\hline BACE1 & - Negative regulator for excitatory synaptic transmission (homeostatic synaptic plasticity) & 15 \\
\hline$\gamma$-secretase/Presenilin & $\begin{array}{l}\text { - Presynaptic short-term plasticity, synaptic facilitation } \\
\text { - Homeostatic synaptic scaling of excitatory synapses }\end{array}$ & $\begin{array}{l}17 \\
18\end{array}$ \\
\hline Tau & - Synaptic stability (presynaptic proteins, synaptic vesicle) & 19,20 \\
\hline ApoE4 & $\begin{array}{l}\text { - Downregulation of amount glutamate } \\
\text { - Modulation of spontaneous vesicle release }\end{array}$ & $\begin{array}{l}27 \\
28\end{array}$ \\
\hline
\end{tabular}


Hence, the production and release of $A \beta$ are modulated by activity-dependent synaptic transmission and endocytosis at the nerve terminals $(10,11)$.

\section{Beta-secretase (BACE)}

$\beta$-site amyloid precursor protein-cleaving enzyme 1 (BACE1) is a key enzyme that produces Amyloid beta under pathological conditions. Localized at the synaptic vesicles, BACE1 is important for synaptic functions. Numerous potential substrates have been identified, which contain several synaptic proteins, in addition to APP (12). Furthermore, BACE1 was biochemically detected in the synaptic vesicle enriched fraction, indicating that synaptic vesicle is the likely location for APP processing $(13,14)$. BACE1 KO mice revealed that basal excitatory synaptic transmission was augmented. It is likely that the pathway downstream of BACE1 at the synapse was decreased, which could be due to the scaling of homeostatic synaptic plasticity (15). Synaptic adhesion protein Neuroligin1 and voltage-gated sodium channel were also known substrates for BACE1; however, it is as yet unknown how BACE1 regulates these substrates.

\section{$\gamma$-secretase and presenilin}

$\gamma$-secretase, along with BACE1, is essential for the production of a 42 peptide of amyloid beta. Several functions of $\gamma$-secretase have been reported at synapses. Localization studies revealed that $\gamma$-secretase is present in the synaptic endosomal fraction of rat brain, which is highly overlapped with the localization of BACE1 protein (16). In neurons with conditional knockout of presenilin, one of the subunits of the $\gamma$-secretase complex, presynaptic short-term plasticity and synaptic facilitation were severely altered, mainly mediated by presynaptic functions; these impairments result from intracellular $\mathrm{Ca}^{2+}$ release at the presynaptic terminals (17). In addition, hippocampal neurons derived from presenilin $\mathrm{KO}$ mice failed to the homeostatic scaling of excitatory synapses (18). Collectively, presenilin regulates neurotransmission at the nerve terminals.

\section{Tau}

Tau was originally discovered as a microtubule-associated protein. The neurofibrillary tangle (NFT) or paired helical filament (PHF) one of the major hallmarks of $A D$, is formed by Tau protein aggregation. However, studies report the functioning of tau at synapses. Tau is involved in axonal transport and synaptic protein stability for regulation of microtubule stability (19). In addition, it also provides the structural support to form and maintain synapses (20). Truncated tau contains specific phosphor-pattern, and can be localized both at the pre- and post-synaptic compartments. Particularly, at the presynaptic terminal, it impairs the stability of microtubules, resulting in a reduction of synaptic vesicles (21).

In pathological conditions, the Tau protein strongly influences synaptic dysfunction. The brain of the $\operatorname{rg} 4510$ mouse, a human mutant P301L tau overexpressed mouse model, revealed age-dependent synaptic loss at both the preand post- synaptic regions, subsequently resulting in synaptic dysfunction. Tauopathy exhibits a strong impairment of synaptic transmission, and in combination with APP models, the synaptic impairment was aggravated, suggesting that the two pathological protein (Tau and APP) act in concert with synaptic function and dysregulation $(22,23)$.

\section{Apolipoprotein (APOE)}

ApoE is a lipoprotein mainly involved in the transport of lipoprotein, cholesterol, and lipid-related materials. It is well known that ApoE is strongly related to the pathology of AD, and is also associated with another AD factor, such as Amyloid-beta. Particularly, the apolipoprotein E4 (ApoE4) allele is the major causative allele of ApoE, having a functional role in nerve terminals. Hippocampal neurons with ApoE4 allele expression have a high sensitivity to environmental factors that lower the levels of presynaptic proteins such as synaptophysin $(24,25)$, although the synaptic area in the dentate gyrus increases (26). In addition, ApoE4 targets the replacement mice, showed down-regulation of glutaminase which converts the glutamine to glutamate, and up-regulation of the vesicular glutamate transporter. Consequently, neurons replaced with ApoE4 release decreased levels of glutamate at nerve terminals (27). Interestingly, this effect on presynaptic terminals appear to be restricted only in ApoE4 alleles, and not in other E2 and E3 alleles, thereby suggesting that structural and functional regulation is specifically influenced by the ApoE4 allele. Recently, it has been discovered that several ApoE receptors (e.g. Apoer2 and Vldlr) are expressed at the nerve terminal membranes. Reelin, a ligand for ApoE receptor, signaled a transient increase of intracellular $\mathrm{Ca}^{2+}$, resulting in elevation of spontaneous vesicle release by VAMP7 mediated fusion (28).

Also, ApoE4 and amyloid beta were closely associated in $A D$ pathogenesis. In a patient with $A D$, the ApoE4 was colocalized with oligomeric $A \beta$, and enhanced the synaptic localization of oligomeric $A \beta$. These findings suggest that ApoE4 is a stimulator for oligomeric $A \beta$ toxicity for synapses (29). The proteomic response in nerve terminals is more susceptible than in the cell body, suggesting that ApoE has a nerve terminal region-specific functional effect.

\section{NERVE TERMINALS IN PARKINSON'S DISEASE}

Parkinson's disease (PD) is the second most common neurodegenerative disorder. It is a movement disorder characterized by bradykinesia, postural instability, and rigidity, following the progressive loss of dopaminergic neurons in the midbrain. Pathogenesis of PD is classified into sporadic and familial cases, which develop due to environmental and genetic factors. About two dozen genetic factors of PD have been identified so far. Few genetic factors, including $\alpha$-synuclein, 
Table 2. Summary of presynaptic phenotype by PD genetic factors

\begin{tabular}{llc}
\hline & \multicolumn{1}{c}{ Parkinson's disease } & Ref \\
\hline \multicolumn{1}{c|}{ Factor } & \multicolumn{1}{c}{ Phenotype at presynaptic terminal } & 38 \\
$\alpha$-synuclein & - Impairment of dopamine release in SNpc & 39,40 \\
& - Reduction of synaptic vesicle endocytosis and reclustering & 41 \\
LRRK2 & - Impairment of release and decreased DA uptake in SNpc & 48 \\
Parkin & - Impairment of synaptic endocytosis in presynaptic terminals & 45 \\
& - Reduction of dopamine release & 55 \\
PINK1 & - Impairment of synaptic plasticity in striatal cells & 53 \\
DJ-1 & - Defect of LTD through inhibitory effects of D2 receptor & 62 \\
Synaptojanin1 & - Slowed endocytosis rate for small stimulation by defect of phosphatase activity & 68 \\
Endophilin & - Regulation of Parkin expression & 74,75 \\
\hline
\end{tabular}

LRRK2 (Leucine-rich repeat kinase 2), Parkin, PINK-1 (PTEN Induced Putative Kinase 1) and DJ-1, have been thoroughly researched in the pathogenesis of PD. Accumulating evidence shows that genetic factors of PD are associated with alteration of the synaptic functions $(30,31)$ (Table 2 ).

\section{$\alpha$-synuclein}

$\alpha$-synuclein is a small protein containing 140 amino acids, and contributes to early-onset PD (32). Generally, $\alpha$-synuclein localizes at the presynaptic terminal. It is associated with synaptic vesicles, controlled synaptic vesicle trafficking, and SNARE complex formations at the nerve terminal (33-35). In pathological conditions, $\alpha$-synuclein is implicated in the alteration of synaptic functions. Human $\alpha$-synuclein overexpressing animal models show protein aggregations at the nerve terminals $(36,37)$, and overexpression of human $\alpha$-synuclein by viral vector injection into substantia nigra in animal models leads to impaired dopamine release (38). Furthermore, neurotransmission inhibition might be related to the impairment of synaptic vesicle endocytosis (39) or synaptic vesicle reclustering after synaptic vesicle endocytosis (40). In addition, the overexpressing pathogenic mutants of $\alpha$-synuclein (A30P and A53T) in primary midbrain neurons, led to abnormal neurite growth and reduced the recycling pool of synaptic vesicles (41). This evidence suggests that $\alpha$-synuclein aggregation alters synaptic formation and functions.

\section{LRRK2}

LRRK2 is a large multidomain protein which includes kinase, GTPase, and protein-protein interaction domains. It is one of the prominent familial PD factors, particularly the gain-offunction mutant of LRRK2 (G2019S) is strongly associated with familial PD as well as sporadic PD $(42,43)$. Several studies report that LRRK2 is implicated in the structural and functional regulation of synapses through kinase-dependent mechanisms. It regulates the presynaptic and postsynaptic morphology by the phosphorylation-dependent interaction of Futsch and $4 \mathrm{E}-\mathrm{BP}$ in fly models (44). LRRK2 participates in synaptic vesicle endocytosis by phosphorylating endophilin (45), which is related with delayed endocytosis of synaptic vesicles, and subsequently affecting the neurotransmission impairment (46). LRRK2 also phosphorylates the NSF (N-ethylmaleimide-Sensitive Factor) D2 domain (Threonine 645), which plays a key role in SNARE complex disassembly after synaptic vesicle exocytosis. NSF phosphorylation by LRRK2 exhibits an elevated rate of SNARE disassembly (47). BAC transgenic animals for LRRK2 G2019S mutation, characterized by elevated kinase activity, show impairment of striatal dopamine release and a decrease of dopamine uptake, without dopaminergic neuron loss in the substantia nigra pars compacta (SNpc) (48). Furthermore, a neuron with LRRK2 G2019S expression shows elevated release probability with increased synaptic density (49), and altered glutamatergic synaptic plasticity (50).

\section{Parkin}

Parkin is an E3 ubiquitin ligase, and has an important role in cellular homeostasis due to its regulation of mitophagy and protein degradation. However, the loss-of-function mutation of Parkin is associated with juvenile-onset PD $(51,52)$. Parkin has been implicated in the modulation of synaptic functions. Parkin KO mice show decrease of evoked dopamine release in the striatum, and the striatal medium spiny neuron exhibit impairments of synaptic plasticity, which are long-term depression and long-term potentiation (53). Parkin also negatively regulates the number and strength of excitatory synapse (54), and neurotransmission is impaired by reduced AMPA receptor endocytosis due to loss of function of Parkin (55). Several studies report that functional loss of Parkin impaired degradation of synaptic proteins, including $\alpha$-synuclein, synphilin- 1 , and CDCrel- 1 , thereby contribute to protein aggregation (56-58). 


\section{PINK1}

Inherited nonsense and missense mutation of PINK1 (PTEN-induced putative kinase1) is a known early-onset familial PD factor (59). PINK1 has a N-terminal mitochondrial targeting motif and a conserved kinase domain (60), and is closely related to mitochondrial function and mitochondrial quality control (61). Pathologic mutation of PINK1 results in abnormal morphology of mitochondria. In addition, there is impairment of dopamine release, which presumably relates to synaptic mitochondrial dysfunction by pathogenic PINK1 (62). Loss of PINK1 also impairs the normal development of dopaminergic neuron, consequently leading to locomotor dysfunction (63). PINK1-deficient mice show normal number of dopaminergic neurons, but the release of dopamine is significantly decreased, suggesting that PINK1 has a role in synaptic transmission (64).

\section{DJ-1}

Generally, DJ-1 acts as a sensor for cellular redox homeostasis (65). However, functional mutation of DJ-1 is a causative familial factor for autosomal recessive early-onset PD (66). Localization studies reveal that DJ-1 is localized in the synaptic membrane. The binding affinity for synaptic membrane reduces with pathogenic DJ-1 compared to WT DJ-1 (67), indicating that it is likely involved in synaptic functions. In fact, DJ-1 depleted mice show signs of LTD (long-term depression), through the inhibitory effects of the D2 receptor by loss of DJ-1 (68).

\section{Synaptojanin-1}

Synaptojanin-1, a known phosphoinositide phosphatase, has a role in endocytosis process. It interacts with several endocytic proteins such as dynamin, Dap160/intersectin, and BAR proteins including endophilin and amphiphysin (69, 70), suggesting a key role in synaptic vesicle recycling processing, particularly clathrin-coated pit uncoating (71). Recently, the Sac1 domain mutation of synaptojanin-1 (p.Arg258Gln) has been reported in a family with early-onset progressive Parkinsonism $(72,73)$. Although synaptojanin-1 mutation mediated pathogenesis of PD has been less explored, the pathogenic phenotype is exhibited in mutations of synaptojanin-1 associated with PD, as well as early onset refractory seizures and neurological decline $(74,75)$, suggesting that the loss-of-function of synaptojanin-1 may contribute to the pathogenesis of PD and other neurological diseases by impaired synaptic vesicle recycling.

\section{Endophilin}

Endophilin is a key factor in synaptic vesicle recycling. Recently, however, some papers report that it is related to PD genetic factors, including LRRK2, parkin, and synaptojanin-1 $(45,76,77)$. Endo-A, a fly ortholog of endophilin, is a substrate for LRRK2. BAR domain (Serine75) in Endo-A is phosphorylated, and recruitment of Endo-A to the endocytic complex during endocytosis gets modulated. Consequently, hyper-phosphorylation of BAR domain of Endo-A in LRRK2 G2019S mutant shows impairment of synaptic endocytosis in presynaptic terminals (45). In addition, endophilin phosphorylation by LRRK2 increases the recruitment of atg3 to the membrane area of presynaptic terminals, resulting in macroautophagy induction, affecting the membrane curvature induction for autophagy (78). Interestingly, endophilin mutant mice exhibited strongly increased parkin expression, suggesting that endophilin genetically interacts with parkin (76).

\section{NERVE TERMINALS IN OTHER NEURODEGENERATIVE DISEASES}

ALS

Amyotrophic lateral sclerosis (ALS) is a motor neuron disorder characterized by progressive loss of motor neurons in the cortex, brainstem and spinal cord. The loss of motor neuron leads to muscle atrophy and weakness, thereby eventually resulting in death. Superoxide dismutase-1 (SOD-1), one of the most prominent ALS genetic factors, is an antioxidant enzyme involved in the conversion of free superoxide radicals to oxygen and hydrogen peroxide. Both, a dominant and a recessive mutation of SOD-1, have been identified in ALS patients (79-81). It has been reported that the mutations of SOD-1 were implicated in synaptic dysfunctions. Both wild type of SOD-1 and pathogenic SOD-1 were localized at the pre and post-synapse. The G93A SOD-1 mutant, one of the pathogenic SOD-1 mutants, shows mislocalization in presynaptic terminals as well as post-synapse, thereby impairing axonal transport and contributing neuronal cell death $(82,83)$. SOD1 mutant mice also show length-dependent axonopathy with synaptic degeneration (84), and decreased synaptophysinpositive presynaptic bouton in the remaining motor neurons (85). Mutation of TDP-43, a DNA-/RNA-binding protein which modulates RNA splicing and micro RNA biogenesis $(86,87)$, has been identified in familial ALS. Transgenic animals of the mutant with human TDP-43 exhibit reduced levels of

Table 3. Summary of presynaptic phenotype by ALS and HD genetic factors

\begin{tabular}{|c|c|c|c|}
\hline \multicolumn{4}{|c|}{ ALS and Huntington's disease } \\
\hline & actor & Phenotype at presynaptic terminal & Ref \\
\hline \multirow[t]{3}{*}{ ALS } & SOD-1 & $\begin{array}{l}\text { - Axonal transport } \\
\text { - Synaptic degeneration }\end{array}$ & $\begin{array}{c}83 \\
84,85\end{array}$ \\
\hline & TDP-43 & $\begin{array}{l}\text { - Expression regulation of presynaptic protein } \\
\text { - Attenuation of synaptic transmission }\end{array}$ & $\begin{array}{l}88 \\
89\end{array}$ \\
\hline & FUS & - Active zone formation, synaptic transmission & 93 \\
\hline HD & $\mathrm{HTT}$ & $\begin{array}{l}\text { - Synaptic transmission, release probability } \\
\text { - Synaptic vesicle dynamics }\end{array}$ & $\begin{array}{c}94 \\
95,96\end{array}$ \\
\hline
\end{tabular}




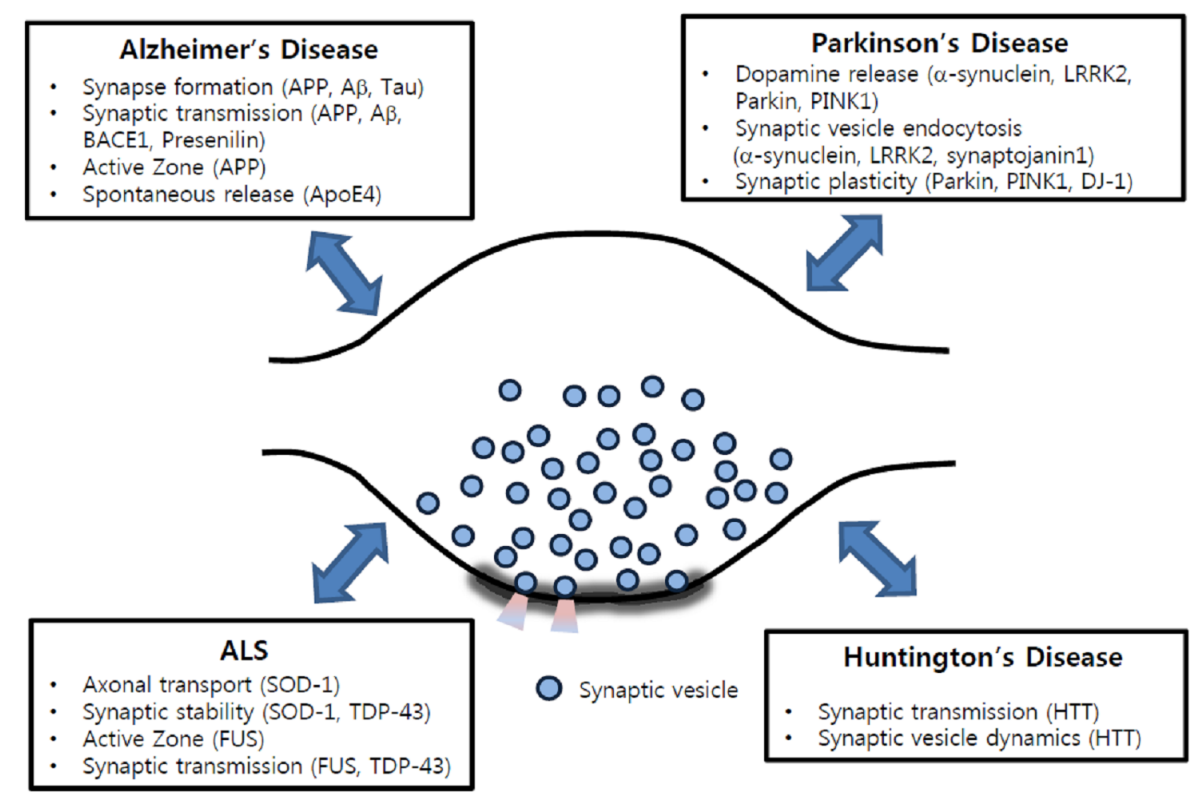

Fig. 1. Summarization of diagram for alteration of presynaptic terminals in various neurodegenerative diseases. synaptophysin (a presynaptic protein) in the brain, as well as cognitive and motor deficits in behavioral tests (88); also, the synaptic transmission was attenuated (89). FUS (Fused-inSarcoma) is also one of the DNA/RNA-binding proteins having a similar structure and functions as TDP-43 (90). The mutation in nuclear localization signal (NLS) of FUS leads to increased cytoplasmic FUS position, which induces the aggregation of FUS mutants, as a pathogenesis of ALS $(91,92)$. FUS mutations were also linked to synaptic dysfunctions. Overexpression FUS mutant disrupts formation of presynaptic active zones, consequently reducing the synaptic transmission with decreased quantal size (93) (Table 3).

\section{Huntington's disease}

Huntington's disease (HD) is an inherited autosomal dominant neurodegenerative disorder. It is mainly caused by mutation of the huntingtin (htt) protein, which has an abnormally high copy of polyglutamine (polyQ) repeat at the $\mathrm{N}$-terminus. General symptoms of HD are motor dysfunction and cognitive deficits, which correlate with the neurodegeneration of specific regions such as the striatum and cerebral cortex. Some of the presynaptic alterations in HD have been reported in various genetic models. An HD model system expressing 128 polyQ expansion in Drosophila, revealed that it had significantly higher neurotransmitter release and release probability (94). Presynaptic specific protein alterations are also reported. For example, rabphilin $3 \mathrm{~A}$ expression level decreased (95); however, levels of SCAMP5, one of the synaptic vesicle proteins was increased (96), suggesting that these alterations of presynaptic protein levels result in an impairment of synaptic vesicle fusion or endocytosis process (Table 3).

\section{CONCLUSION}

We here reviewed the structural and functional alterations of presynaptic terminals by genetic factors in several neurodegenerative diseases (Fig. 1). In AD, APP, an original source for $A \beta$ peptide, is a molecular hub in PAZ. It negatively regulates the nerve terminal formation and readily releasable synaptic vesicle pool. Pathological $A \beta$ (aggregate $A \beta$ ) strongly inhibits the synaptic vesicle fusion machinery; however, soluble $A \beta$ increases the release probability. BACE1 and presenilin are also important regulators for presynaptic physiology. In addition, other genetic factors for AD (Tau and ApoE4) are also involved in synaptic stability and synaptic release. In PD, numerous studies for the genetic factors has revealed the implication in presynaptic functions. $\alpha$-synuclein expression controlled the release probability and recycling pool size, and LRRK2 modulates dopamine release and synaptic vesicle endocytosis by phosphorylating several endocytic proteins (e.g. endophilin). Interestingly, recently accumulating reports show that endocytic proteins (e.g. synaptojanin1, endophilin) are strongly related in PD, indicating that the synaptic vesicle endocytosis process might be an important pathway related in the pathogenesis of PD.

A number of the genetic factors for neurodegenerative diseases are closely related with synaptic function and its alteration. However, most studies just display the phenotype of synaptic dysfunctions, without detailed mechanisms of how the genetic factors result in these dysfunctions. By far, most studies for the pathogenesis of neurodegenerative diseases tend to focus on mechanisms of how neuronal cell death or neurodegeneration occur. Most of the neurodegenerative diseases are generally thought to be chronic diseases. 
Ultimately, neurons are likely to die after experiencing a number of abnormal processes during neurodegeneration. Synapses possess high variability and plasticity, and are also highly vulnerable to pathological conditions. It is likely to reveal abnormal phenotype or alteration of the synaptic function at the early onset of neurodegeneration, suggesting that an in depth investigation for synaptic dysfunction may provide a new approach to the understanding of the early pathogenesis of neurodegenerative diseases.

\section{ACKNOWLEDGEMENTS}

This work is supported by Basic Science Research Program and Brain Science Research Program through the Nation Research Foundation of Korea (NRF) funded by the Ministry of science, ICT \& Future Planning (NRF-2013R1A1A1063174, NRF-2017R1A2B4007019), and a grant from Kyung Hee University in 2016 (KHU-20160702).

\section{CONFLICTS OF INTEREST}

The authors have no conflicting financial interests.

\section{REFERENCES}

1. Ikin AF, Annaert WG, Takei K et al (1996) Alzheimer amyloid protein precursor is localized in nerve terminal preparations to Rab5-containing vesicular organelles distinct from those implicated in the synaptic vesicle pathway. J Biol Chem 271, 31783-31786

2. Groemer TW, Thiel CS, Holt M et al (2011) Amyloid precursor protein is trafficked and secreted via synaptic vesicles. PLoS One 6, e18754

3. Priller C, Bauer T, Mitteregger G, Krebs B, Kretzschmar HA and Herms J (2006) Synapse formation and function is modulated by the amyloid precursor protein. J Neurosci 26, 7212-7221

4. Lassek M, Weingarten J, Wegner M et al (2016) APP Is a Context-Sensitive Regulator of the Hippocampal Presynaptic Active Zone. PLoS Comput Biol 12, e1004832

5. Jang BG, In S, Choi B and Kim MJ (2014) Beta-amyloid oligomers induce early loss of presynaptic proteins in primary neurons by caspase-dependent and proteasomedependent mechanisms. Neuroreport 25, 1281-1288

6. Abramov E, Dolev I, Fogel H, Ciccotosto GD, Ruff E and Slutsky I (2009) Amyloid-beta as a positive endogenous regulator of release probability at hippocampal synapses. Nat Neurosci 12, 1567-1576

7. Fogel H, Frere S, Segev O et al (2014) APP homodimers transduce an amyloid-beta-mediated increase in release probability at excitatory synapses. Cell Rep 7, 1560-1576

8. Romani A, Marchetti C, Bianchi D et al (2013) Computational modeling of the effects of amyloid-beta on release probability at hippocampal synapses. Front Comput Neurosci 7, 1

9. Russell $\mathrm{CL}$, Semerdjieva S, Empson RM, Austen BM, Beesley PW and Alifragis P (2012) Amyloid-beta acts as a regulator of neurotransmitter release disrupting the interaction between synaptophysin and VAMP2. PLoS One 7 , e43201

10. Cirrito JR, Yamada KA, Finn MB et al (2005) Synaptic activity regulates interstitial fluid amyloid-beta levels in vivo. Neuron 48, 913-922

11. Cirrito JR, Kang JE, Lee J et al (2008) Endocytosis is required for synaptic activity-dependent release of amyloidbeta in vivo. Neuron 58, 42-51

12. Munro KM, Nash A, Pigoni M, Lichtenthaler SF and Gunnersen JM (2016) Functions of the Alzheimer's Disease Protease BACE1 at the Synapse in the Central Nervous System. J Mol Neurosci 60, 305-315

13. Lundgren JL, Ahmed S, Schedin-Weiss S et al (2015) ADAM10 and BACE1 are localized to synaptic vesicles. J Neurochem 135, 606-615

14. Del Prete D, Lombino F, Liu X and D'Adamio L (2014) APP is cleaved by Bace1 in pre-synaptic vesicles and establishes a pre-synaptic interactome, via its intracellular domain, with molecular complexes that regulate presynaptic vesicles functions. PLoS One 9, e108576

15. Petrus E and Lee HK (2014) BACE1 is necessary for experience-dependent homeostatic synaptic plasticity in visual cortex. Neural Plast 2014, 128631

16. Frykman S, Hur JY, Franberg J et al (2010) Synaptic and endosomal localization of active gamma-secretase in rat brain. PLoS One 5, e8948

17. Zhang C, Wu B, Beglopoulos V et al (2009) Presenilins are essential for regulating neurotransmitter release. Nature 460, 632-636

18. Pratt KG, Zimmerman EC, Cook DG and Sullivan JM (2011) Presenilin 1 regulates homeostatic synaptic scaling through Akt signaling. Nat Neurosci 14, 1112-1114

19. Spires-Jones TL and Hyman BT (2014) The intersection of amyloid beta and tau at synapses in Alzheimer's disease. Neuron 82, 756-771

20. Voelzmann A, Okenve-Ramos P, Qu Y et al (2016) Tau and spectraplakins promote synapse formation and maintenance through Jun kinase and neuronal trafficking. Elife 5

21. Jadhav S, Katina S, Kovac A, Kazmerova Z, Novak M and Zilka N (2015) Truncated tau deregulates synaptic markers in rat model for human tauopathy. Front Cell Neurosci 9, 24

22. Kopeikina KJ, Polydoro M, Tai HC et al (2013) Synaptic alterations in the rTg4510 mouse model of tauopathy. J Comp Neurol 521, 1334-1353

23. Kopeikina KJ, Wegmann S, Pitstick R et al (2013) Tau causes synapse loss without disrupting calcium homeostasis in the rTg4510 model of tauopathy. PLoS One 8, e80834

24. Levi O, Jongen-Relo AL, Feldon J, Roses $A D$ and Michaelson DM (2003) ApoE4 impairs hippocampal plasticity isoform-specifically and blocks the environmental stimulation of synaptogenesis and memory. Neurobiol Dis 13, 273-282

25. Zhu Y, Nwabuisi-Heath E, Dumanis SB et al (2012) APOE genotype alters glial activation and loss of synaptic markers in mice. Glia 60, 559-569

26. Cambon K, Davies HA and Stewart MG (2000) Synaptic 
loss is accompanied by an increase in synaptic area in the dentate gyrus of aged human apolipoprotein E4 transgenic mice. Neuroscience 97, 685-692

27. Dumanis SB, DiBattista AM, Miessau M, Moussa CE and Rebeck GW (2013) APOE genotype affects the presynaptic compartment of glutamatergic nerve terminals. J Neurochem 124, 4-14

28. Bal M, Leitz J, Reese AL et al (2013) Reelin mobilizes a VAMP7-dependent synaptic vesicle pool and selectively augments spontaneous neurotransmission. Neuron 80, 934-946

29. Koffie RM, Hashimoto T, Tai HC et al (2012) Apolipoprotein E4 effects in Alzheimer's disease are mediated by synaptotoxic oligomeric amyloid-beta. Brain 135, 2155-2168

30. Picconi B, Piccoli G and Calabresi P (2012) Synaptic dysfunction in Parkinson's disease. Adv Exp Med Biol 970, 553-572

31. Belluzzi E, Greggio E and Piccoli G (2012) Presynaptic dysfunction in Parkinson's disease: a focus on LRRK2. Biochem Soc Trans 40, 1111-1116

32. Stefanis L (2012) alpha-Synuclein in Parkinson's disease. Cold Spring Harb Perspect Med 2, a009399

33. Goedert M (2001) Alpha-synuclein and neurodegenerative diseases. Nat Rev Neurosci 2, 492-501

34. Norris EH, Giasson BI and Lee VM (2004) Alphasynuclein: normal function and role in neurodegenerative diseases. Curr Top Dev Biol 60, 17-54

35. Rizo J and Sudhof TC (2012) The membrane fusion enigma: SNAREs, Sec1/Munc18 proteins, and their accomplices--guilty as charged? Annu Rev Cell Dev Biol 28, 279-308

36. Tanji K, Mori F, Mimura J et al (2010) Proteinase K-resistant alpha-synuclein is deposited in presynapses in human Lewy body disease and A53T alpha-synuclein transgenic mice. Acta Neuropathol 120, 145-154

37. Spinelli KJ, Taylor JK, Osterberg VR et al (2014) Presynaptic alpha-synuclein aggregation in a mouse model of Parkinson's disease. J Neurosci 34, 2037-2050

38. Lundblad $M$, Decressac $M$, Mattsson B and Bjorklund A (2012) Impaired neurotransmission caused by overexpression of alpha-synuclein in nigral dopamine neurons. Proc Natl Acad Sci U S A 109, 3213-3219

39. Xu J, Wu XS, Sheng J et al (2016) alpha-Synuclein Mutation Inhibits Endocytosis at Mammalian Central Nerve Terminals. J Neurosci 36, 4408-4414

40. Nemani VM, Lu W, Berge V et al (2010) Increased expression of alpha-synuclein reduces neurotransmitter release by inhibiting synaptic vesicle reclustering after endocytosis. Neuron 65, 66-79

41. Scott D and Roy S (2012) alpha-Synuclein inhibits intersynaptic vesicle mobility and maintains recyclingpool homeostasis. J Neurosci 32, 10129-10135

42. Mills RD, Mulhern TD, Liu F, Culvenor JG and Cheng HC (2014) Prediction of the repeat domain structures and impact of parkinsonism-associated variations on structure and function of all functional domains of leucine-rich repeat kinase 2 (LRRK2). Hum Mutat 35, 395-412

43. Martin I, Kim JW, Dawson VL and Dawson TM (2014) LRRK2 pathobiology in Parkinson's disease. J Neurochem
$131,554-565$

44. Lee $\mathrm{S}$, Liu HP, Lin WY, Guo H and Lu B (2010) LRRK2 kinase regulates synaptic morphology through distinct substrates at the presynaptic and postsynaptic compartments of the Drosophila neuromuscular junction. J Neurosci 30, 16959-16969

45. Matta S, Van Kolen K, da Cunha R et al (2012) LRRK2 controls an EndoA phosphorylation cycle in synaptic endocytosis. Neuron 75, 1008-1021

46. Arranz AM, Delbroek L, Van Kolen K et al (2015) LRRK2 functions in synaptic vesicle endocytosis through a kinase-dependent mechanism. J Cell Sci 128, 541-552

47. Belluzzi E, Gonnelli A, Cirnaru MD et al (2016) LRRK2 phosphorylates pre-synaptic $\mathrm{N}$-ethylmaleimide sensitive fusion (NSF) protein enhancing its ATPase activity and SNARE complex disassembling rate. Mol Neurodegener 11,1

48. Li X, Patel JC, Wang J et al (2010) Enhanced striatal dopamine transmission and motor performance with LRRK2 overexpression in mice is eliminated by familial Parkinson's disease mutation G2019S. J Neurosci 30, 1788-1797

49. Beccano-Kelly DA, Kuhlmann N, Tatarnikov I et al (2014) Synaptic function is modulated by LRRK2 and glutamate release is increased in cortical neurons of G2019S LRRK2 knock-in mice. Front Cell Neurosci 8, 301

50. Beccano-Kelly DA, Volta M, Munsie LN et al (2015) LRRK2 overexpression alters glutamatergic presynaptic plasticity, striatal dopamine tone, postsynaptic signal transduction, motor activity and memory. Hum Mol Genet 24, 1336-1349

51. Leroy E, Anastasopoulos D, Konitsiotis S, Lavedan C and Polymeropoulos MH (1998) Deletions in the Parkin gene and genetic heterogeneity in a Greek family with early onset Parkinson's disease. Hum Genet 103, 424-427

52. Lucking CB, Abbas N, Durr A et al (1998) Homozygous deletions in parkin gene in European and North African families with autosomal recessive juvenile parkinsonism. The European Consortium on Genetic Susceptibility in Parkinson's Disease and the French Parkinson's Disease Genetics Study Group. Lancet 352, 1355-1356

53. Kitada T, Pisani A, Karouani M et al (2009) Impaired dopamine release and synaptic plasticity in the striatum of parkin-/- mice. J Neurochem 110, 613-621

54. Helton TD, Otsuka T, Lee MC, Mu Y and Ehlers MD (2008) Pruning and loss of excitatory synapses by the parkin ubiquitin ligase. Proc Natl Acad Sci U S A 105, 19492-19497

55. Cortese GP, Zhu M, Williams D, Heath $\mathrm{S}$ and Waites $\mathrm{CL}$ (2016) Parkin Deficiency Reduces Hippocampal Glutamatergic Neurotransmission by Impairing AMPA Receptor Endocytosis. J Neurosci 36, 12243-12258

56. Khandelwal PJ, Dumanis SB, Feng LR et al (2010) Parkinson-related parkin reduces alpha-Synuclein phosphorylation in a gene transfer model. Mol Neurodegener 5,47

57. Zhang Y, Gao J, Chung KK, Huang H, Dawson VL and Dawson TM (2000) Parkin functions as an E2-dependent ubiquitin- protein ligase and promotes the degradation of the synaptic vesicle-associated protein, CDCrel-1. Proc 
Natl Acad Sci U S A 97, 13354-13359

58. Chung KK, Zhang Y, Lim KL et al (2001) Parkin ubiquitinates the alpha-synuclein-interacting protein, synphilin-1: implications for Lewy-body formation in Parkinson disease. Nat Med 7, 1144-1150

59. Valente EM, Abou-Sleiman PM, Caputo V et al (2004) Hereditary early-onset Parkinson's disease caused by mutations in PINK1. Science 304, 1158-1160

60. Beilina A, Van Der Brug M, Ahmad R et al (2005) Mutations in PTEN-induced putative kinase 1 associated with recessive parkinsonism have differential effects on protein stability. Proc Natl Acad Sci U S A 102, 57035708

61. Plun-Favreau H, Klupsch K, Moisoi N et al (2007) The mitochondrial protease HtrA2 is regulated by Parkinson's disease-associated kinase PINK1. Nat Cell Biol 9, 12431252

62. Morais VA, Verstreken $P$, Roethig A et al (2009) Parkinson's disease mutations in PINK1 result in decreased Complex I activity and deficient synaptic function. EMBO Mol Med 1, 99-111

63. Xi Y, Ryan J, Noble $S, Y u$ M, Yilbas AE and Ekker $M$ (2010) Impaired dopaminergic neuron development and locomotor function in zebrafish with loss of pink1 function. Eur J Neurosci 31, 623-633

64. Kitada T, Pisani A, Porter DR et al (2007) Impaired dopamine release and synaptic plasticity in the striatum of PINK1-deficient mice. Proc Natl Acad Sci U S A 104, 11441-11446

65. Junn E, Jang $\mathrm{WH}$, Zhao X, Jeong BS and Mouradian MM (2009) Mitochondrial localization of DJ-1 leads to enhanced neuroprotection. J Neurosci Res 87, 123-129

66. Bonifati V, Rizzu P, van Baren MJ et al (2003) Mutations in the DJ-1 gene associated with autosomal recessive early-onset parkinsonism. Science 299, 256-259

67. Usami Y, Hatano T, Imai S et al (2011) DJ-1 associates with synaptic membranes. Neurobiol Dis 43, 651-662

68. Goldberg MS, Pisani A, Haburcak M et al (2005) Nigrostriatal dopaminergic deficits and hypokinesia caused by inactivation of the familial Parkinsonism-linked gene DJ-1. Neuron 45, 489-496

69. Slepnev VI and De Camilli P (2000) Accessory factors in clathrin-dependent synaptic vesicle endocytosis. Nat Rev Neurosci 1, 161-172

70. McPherson PS, Garcia EP, Slepnev VI et al (1996) A presynaptic inositol-5-phosphatase. Nature 379, 353-357

71. Mani M, Lee SY, Lucast L et al (2007) The dual phosphatase activity of synaptojanin 1 is required for both efficient synaptic vesicle endocytosis and reavailability at nerve terminals. Neuron 56, 1004-1018

72. Krebs CE, Karkheiran S, Powell JC et al (2013) The Sac1 domain of SYNJ1 identified mutated in a family with early-onset progressive Parkinsonism with generalized seizures. Hum Mutat 34, 1200-1207

73. Quadri M, Fang M, Picillo M et al (2013) Mutation in the SYNJ1 gene associated with autosomal recessive, early-onset Parkinsonism. Hum Mutat 34, 1208-1215

74. Hardies K, Cai Y, Jardel C et al (2016) Loss of SYNJ1 dual phosphatase activity leads to early onset refractory seizures and progressive neurological decline. Brain 139,
2420-2430

75. Cao M, Wu Y, Ashrafi G et al (2017) Parkinson Sac Domain Mutation in Synaptojanin 1 Impairs Clathrin Uncoating at Synapses and Triggers Dystrophic Changes in Dopaminergic Axons. Neuron 93, 882-896 e885

76. Cao M, Milosevic I, Giovedi S and De Camilli P (2014) Upregulation of Parkin in endophilin mutant mice. J Neurosci 34, 16544-16549

77. Schuske KR, Richmond JE, Matthies DS et al (2003) Endophilin is required for synaptic vesicle endocytosis by localizing synaptojanin. Neuron 40, 749-762

78. Soukup SF, Kuenen S, Vanhauwaert R et al (2016) A LRRK2-Dependent EndophilinA Phosphoswitch Is Critical for Macroautophagy at Presynaptic Terminals. Neuron 92, 829-844

79. Felbecker A, Camu W, Valdmanis PN et al (2010) Four familial ALS pedigrees discordant for two SOD1 mutations: are all SOD1 mutations pathogenic? J Neurol Neurosurg Psychiatry 81, 572-577

80. Robberecht W, Aguirre T, Van den Bosch L, Tilkin P, Cassiman JJ and Matthijs G (1996) D90A heterozygosity in the SOD1 gene is associated with familial and apparently sporadic amyotrophic lateral sclerosis. Neurology 47, 1336-1339

81. Andersen PM (2006) Amyotrophic lateral sclerosis associated with mutations in the $\mathrm{CuZn}$ superoxide dismutase gene. Curr Neurol Neurosci Rep 6, 37-46

82. Lee DY, Jeon GS, Shim YM, Seong SY, Lee KW and Sung JJ (2015) Modulation of SOD1 Subcellular Localization by Transfection with Wild- or Mutant-type SOD1 in Primary Neuron and Astrocyte Cultures from ALS Mice. Exp Neurobiol 24, 226-234

83. Bae JR and Kim SH (2016) Impairment of SOD1-G93A motility is linked to mitochondrial movement in axons of hippocampal neurons. Arch Pharm Res 39, 1144-1150

84. Tallon C, Russell KA, Sakhalkar S, Andrapallayal N and Farah MH (2016) Length-dependent axo-terminal degeneration at the neuromuscular synapses of type II muscle in SOD1 mice. Neuroscience 312, 179-189

85. Zang DW, Lopes EC and Cheema SS (2005) Loss of synaptophysin-positive boutons on lumbar motor neurons innervating the medial gastrocnemius muscle of the SOD1G93A G1H transgenic mouse model of ALS. J Neurosci Res 79, 694-699

86. Gregory RI, Yan KP, Amuthan G et al (2004) The Microprocessor complex mediates the genesis of microRNAs. Nature 432, 235-240

87. Xu ZS (2012) Does a loss of TDP-43 function cause neurodegeneration? Mol Neurodegener 7, 27

88. Medina DX, Orr ME and Oddo S (2014) Accumulation of C-terminal fragments of transactive response DNA-binding protein 43 leads to synaptic loss and cognitive deficits in human TDP-43 transgenic mice. Neurobiol Aging 35, 79-87

89. Handley EE, Pitman KA, Dawkins E et al (2016) Synapse Dysfunction of Layer V Pyramidal Neurons Precedes Neurodegeneration in a Mouse Model of TDP-43 Proteinopathies. Cereb Cortex 1-18

90. Nolan M, Talbot K and Ansorge O (2016) Pathogenesis of FUS-associated ALS and FTD: insights from rodent 
models. Acta Neuropathol Commun 4, 99

91. Da Cruz S and Cleveland DW (2011) Understanding the role of TDP-43 and FUS/TLS in ALS and beyond. Curr Opin Neurobiol 21, 904-919

92. Lagier-Tourenne C and Cleveland DW (2009) Rethinking ALS: the FUS about TDP-43. Cell 136, 1001-1004

93. Machamer JB, Collins SE and Lloyd TE (2014) The ALS gene FUS regulates synaptic transmission at the Drosophila neuromuscular junction. Hum Mol Genet 23, 38103822

94. Romero E, Cha GH, Verstreken P et al (2008) Suppression of neurodegeneration and increased neurotransmission caused by expanded full-length huntingtin accumulating in the cytoplasm. Neuron 57, 27-40

95. Deak F, Shin $\mathrm{OH}$, Tang J et al (2006) Rabphilin regulates SNARE-dependent re-priming of synaptic vesicles for fusion. EMBO J 25, 2856-2866

96. Parker JA, Metzler M, Georgiou J et al (2007) Huntingtininteracting protein 1 influences worm and mouse presynaptic function and protects Caenorhabditis elegans neurons against mutant polyglutamine toxicity. J Neurosci 27, 11056-11064 